\title{
Smart Monitoring of Electric Meters using IoT
}

\author{
P.Kalpana ${ }^{1}$, B.Manju ${ }^{2}$ \\ Post Graduate Student, Department of Computer Sciences and Engineering, R.V.S Technical Campus, Coimbatore, \\ India $^{1}$
}

Assistant Professor, Department of Computer Sciences and Engineering, R.V.S Technical Campus, Coimbatore, India ${ }^{2}$

\begin{abstract}
The technology is now going upwards everywhere in the globe with the tendency to rise even more. But the major backend support required for most of this technology is power supply. So, electricity is now becoming more and more important day by day. Even though the technology is trying to get rid of it, the rate of necessity is currently high. One of the problems faced by the electricity board is taking the reading from the meter to identify the usage of a customer. so a smart monitoring system that will help the electricity boards to fetch the readings of various houses and commercial buildings with the help of internet of technologies, without any intermediation, that makes the interaction possible between the customer and the electricity board through direct communication with mobile application and website and wireless network connectivity attached with Arduino board. Even the customers will be able to know how much power they are consuming so that the awareness among the customers can be developed which may help the nation to use the power carefully. Even we can find the faults, if any, in the meter. Through this, the aim of also conserving budgets regarding the government can be fulfilled by finding faulty meters and avoiding human errors.
\end{abstract}

Keywords: Cognitive Sensor, Energy Efficient, Reliable, Cost-effective.

\section{INTRODUCTION}

In India, almost power consumption is a big crisis as the sources for power generation are being almost utilized. So the current step that the government is taken regarding the issue is to create awareness among the people about the value of it. Even though many houses in the rural areas are still in darkness, the power consumption value has no change. State government, in 2014 when identified this issue, called for an hour power cut for almost 6 months in Kerala. But there are many other problems that the government haven't kept eye on.

The first and foremost things are the faulty meters that show wrong reading to the reading man even if the household appliances consumed a lot of power. The reading man just notes down the reading shown in the meter and generate the bill. So if a 100 houses like this are there in our area means there is a huge loss to the government. But the actual amounts of such meters are unpredictable because the electricity board is not doing regular check-ups to get rid of such issues. At the same time, there are complaints available about over running of some of the meters. There are even cases where these issues remain unhandled. In this case there is a huge loss for the society because they are paying more than for what they have used. To avoid such problems, the introduction of technology to the scene is becoming mandatory in every problem. So with the help of internet, a smart monitoring system for the electric meter which helps to fetch reading automatically to the board using the hardware attached will do the job automated without any intermediate necessary and the board can directly know the usage of each consumer through the interface.

\section{RELATED WORKS}

\subsection{The Person Notices The Meter And Generates Bill Through Mini Biller}

The representative from the authority will visit each of the houses and the commercial buildings under their respective division. They will check each of the meter and use their scenario of calculation to gather the power usage by each meter and they will calculate the amount for the power usage. Different charges are made applicable according to the purpose of usage and the charges are divided into commercial charge and domestic charge after that he will generate the electricity bill for each consumer and distribute it to the consumers. The consumer by getting the bill will visit the EB office or use the online payment to pay the bill. Certain additional charges will be provided as fine if there happens a late bill payment. The authority will get each consumer's bill payment get updated once it is paid. If the due date is over , the representative from the authority will approach those consumers and terminate the power supply by removing the fuse and it will be returned once bill is paid. 


\subsection{Wireless Iot Based Metering System For Energy Efficient Smart Cites}

The design provides a low power consumption smart metering system. This design is implemented at two ends, one on the consumer end for IoT operation and other on the service provider end for managing customers" data through an implemented Website and customized database. Android application is also designed to have a more easily operated system at the customer end.

It is designed to contribute in establishing energy efficient smart cities. Its main aim is to minimize the rapid increase in average electricity consumption issue. Additionally, it provides a more convenient solution to problems faced from using the existing meters, such as the manual readings"e errors from the analog electromechanical meter. The conventional electromechanically post-paid metering system, where the consumer is charged on a monthly basis, provides no sense of the energy used until the end of the month. However, it guarantees that customers will always have electricity supplies, except during blackouts.

Prepaid meters offer many advantages both to the utility provider and the consumers. To the utility provider, this reduces many issues tremendously arising from meter readers such as delays, wrong and infrequent meter reading resulting in bulk amount of billing that consumers would need to pay and further consequent in not paying, disputes and so forth. Additionally, prepayment metering system, encourages users to control their energy consumption, in order to avoid cut off, due to zero credit. Thus, the metering system uses the prepayment metering as an attempt for building energy efficient new cities.

\subsection{Internet Of Things (IoT) Based Energy Meter}

Non-android users can monitor and pay their bills online. The system is more reliable and accurate reading values are collected from energy meters. Live readings of the energy meter can be viewed through Android application. Also, the readings can be viewed online. The human intensive work is avoided and all the values are maintained in the central server. The communication medium is secure and tampering of energy meters can be identified easily. If an error occurs in the system, the value in the central server will not be updated. Once the value updated crosses the threshold time, the server can determine that something is wrong in the system and can report the engineers in EB. Thus, identification of error becomes easier. Since the values are stored in the central database, the reports are made accessible from anywhere in the world. Also, the server is online $24 x$. But its website design is so poor.and PIC microcontroller is used here.

\subsection{Internet Of Things Based Smart Electricity Meters}

Smart meter is one of the applications of IoT. It records the consumption and sends the readings to the utility office on regular basis for monitoring and billing. For a long time, traditional electromechanical meters have been used.

Meter readings were noted down on the monthly basis. But now with the evolution of smart electricity meters, things are changing. Benefits of smart meter over traditional electromechanical meters:

1. Smart meters are less error prone. Accurate readings are obtained by the customers and utility providers.

2. Readings can be sent remotely over the web to the utility providers. Employees need not to be physically present at the site.

3. Tampering of these meters can be easily detected by the authorities.

4. Smart meters when programmed with home appliances can be used to control the electricity consumption.

\section{PROPOSED APPROACH}

A new technically automated system for the monitoring of electric meters and alerts using the internet of technology is being implemented. A hardware setup which consists of an Arduino board, voltage and current sensors, step-down transformer, Atmega328 micro controller, opto coupler, Wi-Fi module and the GSM board collects the data from the electric meters that is attached. So that the meter reading will be used to generate the useful information by the micro controller. This micro controller will be used to send the data to the default address which has been set as the server using the wireless network connectivity .

The data are able to be viewed by the user and authority through the developed websites for the user side and the authority side respectively. Each of the user will have separate credentials available. The website will display the total amount of the current used, the total amount for the power used, and also the total unit of charge. This system will bring the general awareness among the consumers about how much current they are using a day. With this system the wastage of power can be avoided.

For this, a mobile application is also developed. Since the smart phone utilization is increasing, an application to monitor their power used and to notify the customer about the comparison for the power used for certain interval. 
Even if the network is not available, the GSM module will send SMS to the user's mobile number for the certain interval of time also.

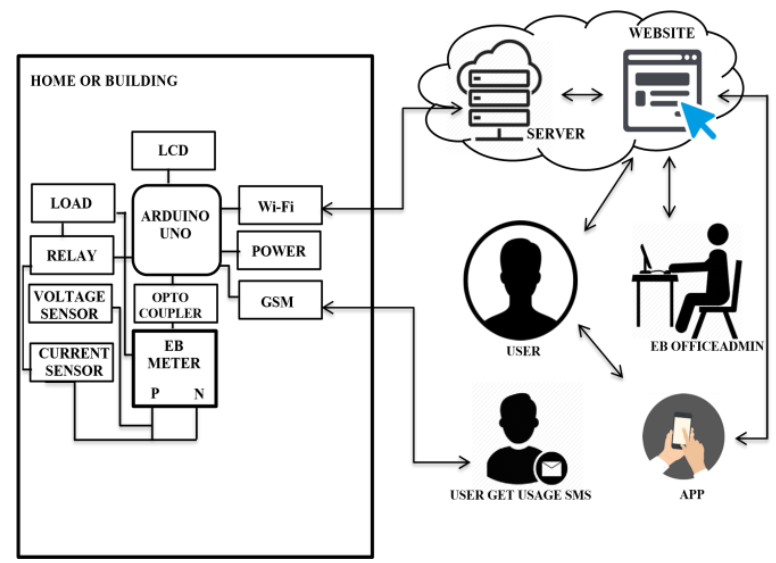

Fig 3.1 Structure of Monitoring System

\subsection{Connection With The Circuit}

To comprises of the hardware implementation on the first. The values are read by the electric meter from the circuit and the data is passed on to the Arduino board by making it pass through the coupler. The Arduino board is the component where the raw data received can be manipulated into a form useful for the system. The GSM module also collects the reading of electricity usage and Send the information to the corresponding user.

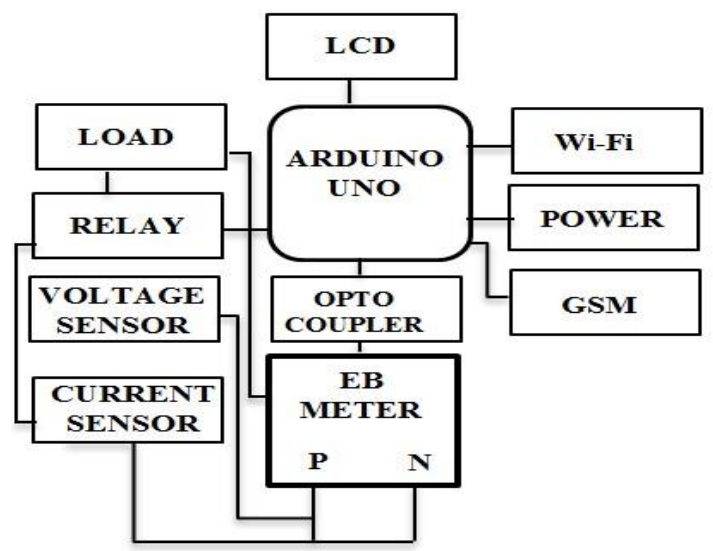

Fig 3.2 Circuit connection

\subsection{Website For EB Office}

The website fetches the useful information from the Arduino board and integrating it with the useres respective web pages for detection and usage verification. Once the data has been fetched, it will be stored into the webpage of each user. The admin can access the entire user's usage of electricity at any time without any high human efforts. If there is any Electric meter fault, then it will be known by admin because the variations in power usage and the faulty meter not update the data on server.

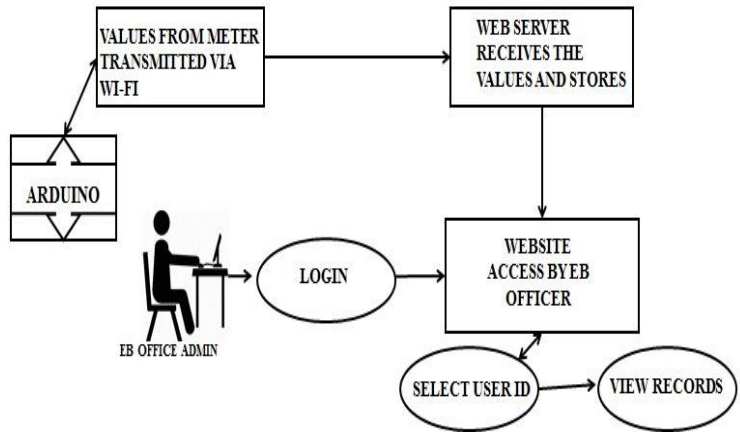

Fig 3.3 Process of EB Office 
Vol. 8, Issue 2, February 2019

\subsection{Website For User}

The module 3 comprises of the development of a website to fetch the useful information from the Arduino board and integrating it with the user"s respective web pages for detection and usage verification. Once the data has been fetched, it will be stored into the webpage of each user. The user with respective id can login to the webpage to see the amount of utilisation of the power which they consumed. Then the user can pay the electricity bill through the online payment option.

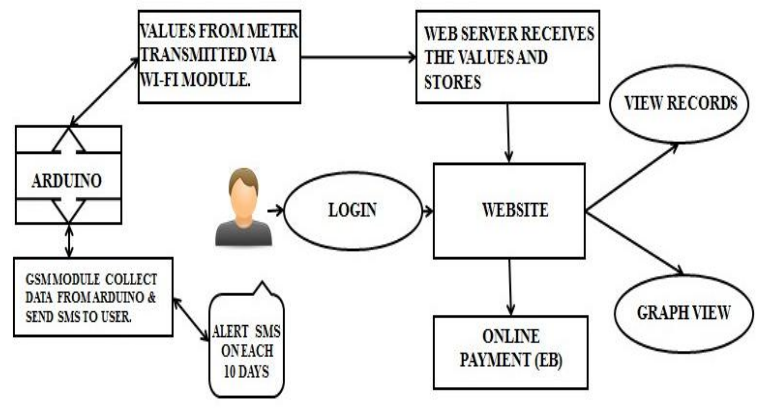

Fig 3.4 Website for EB Office

\subsection{Mobile Application}

The module 4 implements the development of a mobile application so that their power consumption through their mobile. As smart phones are there available with most of the population in the earth, we are utilizing this device to make things easier at the tip of their fingers. Using this application, the user can also check their power consumption and also notification will be given by the application to the users about their power usage and make awareness to the customers.

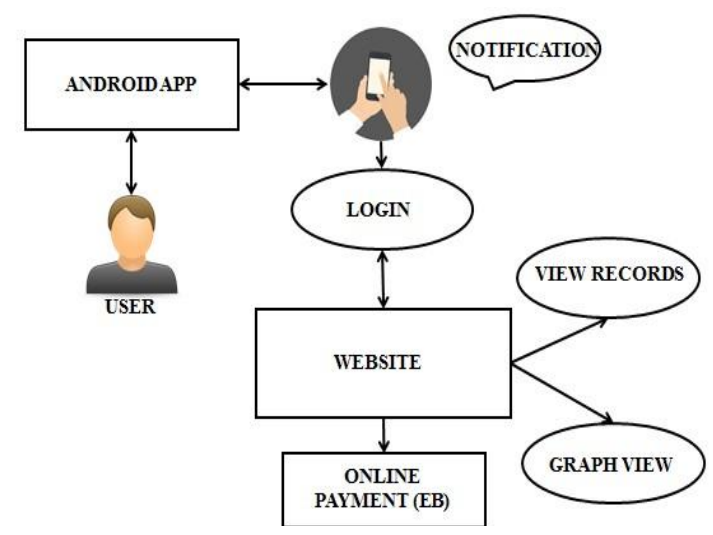

Fig 3.5 Mobile Application

\section{IMPLEMENTATION}

The implementation of the system initiates with the setup of the electric circuit. The circuit will require the Arduino board, Wi-Fi module, voltage and current sensors, GSM module, LCD display, external power supply, opto coupler, step down transformer, ATMEGA 328 micro controller, network connectivity(Wi-Fi) and an electric meter. The circuit can be setup with the electric meter attached to each of the houses and the commercial buildings. The Arduino board is used to collect information from the attached devices and convert it into useful data. The readings from the electric meter will pass through the step down transformer to reduce the 240v supply of power to be transferred to withstand the capability of the other devices to avoid oversupply of power which may result in the damage of the devices and then it is passed through the voltage sensor and current sensor to check monitor the amount of voltage and current supplied. The reduced power will move to the Arduino board through the opto coupler . this opto coupler will reduce the supply further in the sense that it will not destroy or damage the board and other devices attached. The supply received will be converted into the form of data and then it will function as programmed using the IDE.

The data is read by the reader on entering into its area of access. The reader's data is processed by the Arduino board and the data is collected by the microcontroller in it. The data is neither sent nor processed; it is just collected on to the board. Dynamically, the values from the board are sent through an Ethernet module integrated with the Arduino board. 
The Ethernet allows the board to send the values through a medium of Ethernet like internet or to the administrator system.

The system consists of values been authorized first and sent to the server address which is already assigned in the ESP Wi-Fi module. The power supply used then will be continuously received by the micro controller. Then the Wi-Fi module in the circuit will try to get connected to the Wi-Fi network that is available in the nearby areas and then this data stored by the controller will be periodically updated to the server id that has been set as the default destination. The website developed to visualize the utilization of the power by each user is connected to the server and each user will be provided separate credentials to login and see their usage of electricity. Even if the internet support is not available, the GSM module in the circuit will send the data to the user through an SMS to their mobile.

This GSM module will be programmed to send the data in a periodic interval of time. So that even if the network was able to connect to the internet or not, GSM module will send the data to the user's registered mobile number. We know that government is trying to make awareness among the consumers to consume the electricity without wasting it. Through the SMS system, the consumers will come to know about their way of usage and wastage of electricity. Then also through the developed website the authority and the users can check their usage and their total bill to be paid. The authority will have the overall view as admins do; the authority will check each of the consumer with their consumer ID.

So the authority will have the opportunity to know the faulty meters and also be able to avoid the human errors made at the time of calculations of bill for the consumer. Also the mobile application developed can give notification to the customers about their usage in a regular interval of time. The application can be used to know the consumption of the power like through the websites. As smart phones are available everywhere this application can be easily utilized for the monitoring of power consumption.

\section{CONCLUSION}

Due to the human errors and their money saving mentality, the benefits to the government in the case of electricity is still lying hidden under the mysteries. So a system in which no malfunctioning can be performed is essential in our country. This system have been implemented that, automated system in which the perfect readings without human error and also the defective meters can be identified along with other technologies to enhance the security.

\section{FUTURE ENHANCEMENT}

This system can be further implemented completely without the network so that this system can even get implemented in rural areas. Further, the system can be enhanced using some other method to provide immediate action when sudden accidents occurred in a home. The most important phenomenon is usage of cloud integration technologies which can prove to be very scalable and adaptable in future. Along with all the above, the highly configured devices can be made which can provide better performance.

\section{REFERENCES}

[1] Maha Aboelmaged, Yasmeen Abdelghani "Wireless IoT based Metering System for Energy Efficient Smart Cites", 29 ${ }^{\text {th }}$ International Conference on Microelectronics (ICM), 2017.

[2] Gobhinath.S, Gunasundari.N, Gowthami.P “ Internet of Things (IOT) Based Energy Meter”, International Research Journal of Engineering and Technology (IRJET), Vol.03 Issue: 04 ,Apr-2016

[3] Shikha Rastogi, Manisha Sharma and Pratibha Varshney. "Internet of Things based Smart Electricity Meters", International Journal of Computer Applications, vol. 133. Issue 8, January 2016.

[4] Au Thien Wan, Suresh Sankaranarayanan and Siti Nurafifah Binti Sait."Smart Agent Based Prepaid Wireless Energy Meter". International Conference on Cloud Computing and Internet of Things, pp. 77-81. December 2014.

[5] R. Morello, C. De Capua,G. Fulco, S.C. Mukhopadhyay."A Smart Power Meter to Monitor Energy Flow in Smart Grids", IEEE Sensors Journal.2017.

[6] V.Preethi, M.Tech and G. Harish. "Design and Implementation of Smart Energy Meter". International Conference on Inventive Computation Technologies (ICICT), Coimbatore, India. August 2016.

[7] R. MorelloC. De Capua, G. Fulco, S.C. Mukhopadhyay "A Smart Power Meter to Monitor Energy Flow in Smart Grids", DOI 10.1109/JSEN.2017.2760014, IEEE Sensors Journal.

[8] Mohammed Reyasudin Basir Khan and Razali Jidin. "USB Data Acquisition Device for Energy Monitoring," presented at Student Conference on Research and Development, Selangor, Malaysia, 2011. 